\section{A relação médico-paciente no Programa Saúde da Família: um estudo em três municípios do Estado da Bahia, Brasil}

\author{
The physician-patient relationship \\ under the Family Health Program in three \\ municipalities in Bahia State, Brazil
}

\author{
1 Universidade Católica de \\ Salvador, Salvador, Brasil. \\ 2 Instituto de Saúde Coletiva, \\ Universidade Federal da \\ Bahia, Salvador, Brasil. \\ Correspondência \\ A. L. S. Franco \\ Centro de Pesquisa e \\ Extensão, Universidade \\ Católica de Salvador. Rua \\ Ilhéus 205, Salvador, BA \\ 41940-570, Brasil. \\ anameliafranco@uol.com.br
}

\section{Abstract}

This study discusses the physician-patient relationship under the Family Health Program (FHP) in Bahia State, Brazil. Assuming that the physician-patient relationship is influenced by macro and micro-contextual factors, we linked health surveillance and the ecological model of human development, both influenced by contextualism. The methodological strategies included: a census of FHP physicians in Bahia and direct observation of consultations by 20 physicians in three municipalities (counties). There were significant differences in the implementation of the FHP in the three municipalities, with an impact on the physician-patient relationship. As for orientation of clinical practice, health surveillance has not been incorporated by the physicians observed in this study. According to the micro-contextual analysis, health problems were frequently not treated in a contextualized way. FHP guidelines, when incorporated by the municipalities, produce differences in care as observed in the physician-patient relationship. The health surveillance approach, reported as a tool for collective work, proves efficient for describing, evaluating, and improving the FHP, and this study concludes that it is a powerful conceptual tool for dealing with the physician-patient relationship.

Physician-Patient Relations; Family Health Program; Public Health Surveillance
Anamélia Lins e Silva Franco 1 Ana Cecília de Sousa Bastos 2 Vânia Sampaio Alves 2

\section{Introdução}

A relação médico-paciente é um tema de interesse para a Saúde Pública, particularmente para o contexto do Programa Saúde da Família (PSF), que, enquanto estratégia de reorientação do modelo assistencial, pressupõe mudanças na relação entre os profissionais de saúde e os usuários. De acordo com as diretrizes dessa estratégia, espera-se que o médico valorize a relação com os pacientes e as famílias, compreendendo essa relação como parte de um processo terapêutico 1 .

A revisão de estudos desenvolvidos, principalmente no nível da atenção básica à saúde, constata significativa produção sobre a relação médico-paciente. Entretanto, esta literatura tem se restringido muitas vezes à compreensão dessa relação com base em aspectos comunicacionais. No que diz respeito às estratégias metodológicas destes estudos, muitos deles enfocam a relação médico-paciente baseando-se em dados coletados a posteriori das consultas 2 . Outra tendência é de que os estudos, com poucas exceções, são baseados em check-lists, construídos com a finalidade limitada de elaborar tipologias 3,4,5,6 .

Para facilitar a compreensão destas considerações pode-se tomar como exemplo o estudo de Roter et al. 4 , sobre a relação médico-paciente, com o objetivo de identificar padrões na prática médica por meio de dados empíricos. Foram analisadas 537 consultas de 127 médicos, 
tendo médicos e pacientes respondido, imediatamente após a consulta, um questionário que media a satisfação. Os autores identificaram cinco padrões da relação médico-paciente: Biomédico Superficial, Biomédico Extensivo, Biopsicossocial, Psicossocial e Consumidor. O estudo, entretanto, não contemplava o contexto em que ocorria a consulta médica.

A influência do contexto na relação médico-paciente foi observada no estudo de Sucupira 7, que investigou essa relação na clínica pediátrica em três modalidades assistenciais: $o$ centro de saúde da rede estadual, os serviços próprios do Instituto Nacional de Assistência Social e os serviços de uma unidade de "medicina de grupo". A autora verificou diferenças na relação médico-paciente segundo a modalidade assistencial. Apesar da relevância desta proposição, poucos estudos sobre o tema têm privilegiado a dimensão contextual.

A possibilidade de tratar a relação médicopaciente de forma contextualizada pode encontrar, na abordagem ecológica do desenvolvimento proposta por Bronfenbrenner 8, um referencial. Franco \& Bastos 9 revisaram essa abordagem e propuseram uma reflexão da relação médico-paciente sob influência de fatores contextuais, utilizando os conceitos de micro e macrocontexto.

O microcontexto pode ser compreendido como um padrão de atividades, papéis e relações interpessoais constituintes de uma díade primária, caracterizada por sua existência em condições físicas ou fenomenológicas. O macrocontexto inclui elementos culturais e políticos orientadores de práticas grupais e institucionais, a exemplo dos princípios e diretrizes do PSF. Assumindo o pressuposto de que o micro e o macrocontexto repercutem na relação médico-paciente, este estudo teve como objetivo geral compreender essa relação no PSF levando em consideração dimensões micro e macrocontextuais.

\section{Metodologia}

A estratégia metodológica do estudo pretendeu superar limites de trabalhos anteriores ao construir um objeto e respectiva coleta e análise de dados contemplando aspectos contextuais, a exemplo da diversidade relativa à implantação do PSF nos municípios e atuação do médico em zonas urbana e rural, o registro in loco e em tempo real da relação médico-paciente.
Inicialmente, realizou-se um estudo extensivo, de caráter censitário, com utilização de entrevistas telefônicas. O censo do médico de saúde da família da Bahia, Brasil, reuniu dados sócio-demográficos e possibilitou aprofundar o conhecimento da rotina do profissional junto ao paciente 10 . Posteriormente, realizou-se um estudo observacional em três municípios baianos com participação de 20 médicos, dos quais 13 eram mulheres. Cada profissional teve, em média, vinte consultas observadas e gravadas em áudio, sob consentimento informado dos profissionais e pacientes.

Os municípios nos quais realizou-se o estudo foram selecionados com base em critérios geográficos: o Município I, da região metropolitana; o Município II, do semi-árido baiano e o Município III da região litorânea.

Realizaram-se três perspectivas de análise: (i) análise observacional; (ii) análise temática; e (iii) análise interacional. A primeira análise foi orientada por um instrumento observacional 11, desenvolvido para esta finalidade, aplicado ao longo das consultas. Para a análise temática, identificaram-se, a partir do estudo documental das diretrizes do PSF, sete categorias temáticas: natureza do PSF, vigilância da saúde, compromisso com a população adscrita, trabalho em equipe, família como unidade de trabalho, característica da atenção e paciente como sujeito. Para cada categoria foram identificados episódios que representam oportunidades não aproveitadas de exercício das diretrizes. Esta análise realizou-se mediante a transcrição das consultas de dez médicos. A análise interacional buscou caracterizar a relação médico-paciente baseando-se nas consultas de quatro profissionais: uma médica do município da região metropolitana de Salvador, Bahia; um médico da zona rural e uma médica da zona urbana do município da região do semi-árido; e um médico da zona urbana do município da região litorânea.

\section{O macrocontexto: caracterização dos municípios}

O Município I, da região metropolitana, possuía 113.543 habitantes, sendo 5.158 da zona rural (Censo Demográfico 2000; http:/ / www.ibge.gov. br, acessado em 14/Set/2002). O PSF foi implantado em 1998. No período da coleta dos dados, o município contava com quatro equipes. Estas trabalhavam em duas unidades que não 
eram exclusivas do PSF, pois abrigavam atividades orientadas pela demanda espontânea. Isto era motivo de dificuldades de compreensão por parte dos usuários e dos profissionais que não pertenciam à equipe do PSF a respeito das suas diretrizes.

As equipes privilegiavam atendimentos ambulatoriais na comunidade, como escolas e igrejas. Os médicos trabalhavam articulados com os agentes comunitários. Um desafio para o PSF era a desarticulação do sistema de saúde local, fazendo com que o usuário necessitasse encontrar os meios para execução dos exames solicitados, como também a insuficiência da assistência farmacêutica.

O Município II, do semi-árido baiano, possuía 262.494 habitantes, 36.949 na zona rural (Censo Demográfico 2000; http://www.ibge.gov. br, acessado em 14/Set/2002). O município implantou o PSF desde 1998, possuindo o maior número de equipes implantadas no estado na ocasião da coleta de dados, bem como as equipes mais antigas. As equipes do PSF trabalhavam, em sua maioria, em unidades que abrigavam duas equipes, contando ainda com instalações comuns de consultório odontológico, sala de vacinação, de curativos e de nebulização. O sistema de saúde local dispunha de laboratório próprio e um centro municipal de assis tência especializada. Neste, alguns dos médicos do PSF atuavam segundo sua especialidade médica em horários noturnos. Este município apresentava uma rede de serviços de saúde integrada que favorecia o funcionamento da referência e contra-referência. Os medicamentos prescritos freqüentemente eram entregues pelo próprio médico no ato da prescrição.

A distribuição das equipes contemplava o atendimento nas zonas urbana e rural. Para as equipes da zona rural, contava-se com veículo oficial para deslocamento dos profissionais. As equipes realizavam rotineiramente atividades ambulatoriais, visitas domiciliares e grupos educativos.

O Município III, da região litorânea, contava com 95.721 habitantes, 16.102 na zona rural (Censo Demográfico 2000; http://www.ibge.gov. br, acessado em 14/Set/2002). Na ocasião da coleta dos dados, situava-se entre aqueles que possuíam maior número de equipes no estado, tendo a implantação destas iniciado em 1999. Estas atuavam em unidades próprias que, em algumas situações, acolhiam duas equipes. $\mathrm{O}$ município possuía um sistema de referência e contra-referência que poderia ser caracterizado como deficiente. $\mathrm{O}$ acesso a outros níveis de complexidade da assistência por meio do PSF, que deveria ser a "porta de entrada" no siste- ma, não estava garantido. A distribuição de medicamentos era centralizada em uma farmácia que se encontrava fechada comprometendo a assistência farmacêutica. $O$ trabalho em equipe e visitas domiciliares não eram referidos, e o trabalho na zona rural era dificultado pela irregularidade da locomoção. A maioria dos médicos exercia atividades no hospital geral em regime de plantão.

\section{Aproximação ao microcontexto: censo sócio-demográfico do médico do PSF no Estado da Bahia}

A realização do censo justificou-se pela inexistência de registros ou informações acerca do médico do PSF no estado até o ano 2000. Participaram do censo $89,7 \%$ dos profissionais que atuavam nos municípios da Bahia no período.

Quanto ao sexo, os médicos dividiam-se igualmente entre homens e mulheres, dos quais $36,5 \%$ tinham idade entre 24 e 30 anos, e 18,8\% entre 46 e 50 anos. Quanto ao estado civil, mais da metade era casada e $32,3 \%$ solteiros.

Os principais relatos a respeito dos motivos de ingresso no PSF foram a identificação com o programa e com o trabalho em comunidade $(76,1 \%)$; a oportunidade de emprego e o retorno financeiro (23,9\%); razões pessoais, como o desejo de morar em determinado município (15,7\%); identificar-se ou ser identificado com o perfil esperado para o PSF (12,5\%); e a possibilidade de ter uma melhoria de qualidade de vida $(10,5 \%)$.

Com relação à formação acadêmica, faz-se necessário o esclarecimento de que o Estado da Bahia contou por quarenta anos, aproximadamente, com dois cursos de graduação médica: o da Universidade Federal da Bahia (UFBA) e o da Escola Bahiana de Medicina e Saúde Pública (FBDC/EBM), esta mantida por uma Fundação de natureza privada. Um dos elementos que distingue os dois cursos diz respeito à ênfase clínico-laboratorial, orientadadora da Escola Bahiana. O censo possibilitou saber que $49,0 \%$ dos médicos tinham se graduado na FBDC/EBM, enquanto que $19,8 \%$ na UFBA, $15,6 \%$ fizeram sua formação no Sudeste do país e 13,5\% se graduaram em alguma outra faculdade do Nordeste.

Em consonância com o perfil jovem do médico do PSF, 40,6\% tinham menos de 5 anos de formado, 34,4\% tinham entre 6 e 15 anos e $25,0 \%$ mais de 15 anos. Apenas $34,4 \%$ cursaram a residência médica, tendo sido identificadas especialidades diversas: saúde pública ou coletiva $(7,3 \%)$, pediatria $(5,2 \%)$, clínica médica $(4,2 \%)$, ginecologia e obstetrícia $(3,1 \%)$ e outras 
especialidades como anestesiologia, pneumologia e cardiologia (13,5\%).

Dentre as atividades, o atendimento ambulatorial foi referido como prática principal, com $46,9 \%$ dos médicos atendendo de quatro a seis turnos e $44,7 \%$ de sete a dez. Com relação ao número de consultas por turno de atendimento, $54,2 \%$ dos médicos relataram atender de 16 a 20 pacientes por turno; $21,9 \%$ de 11 a 15 por turno; $15,6 \%$ mais de 20 ; e $4,2 \%$ de 8 a 10 pacientes por turno. Em algumas equipes, além dos pacientes agendados, permanecia o atendimento por demandas espontâneas. Com relação à visita domiciliar, 55,2\% dos médicos faziam visita entre um e três turnos na semana; $19,8 \%$ entre quatro e cinco; $2,1 \%$ entre seis e sete; e $19,8 \%$ relataram não possuir um horário especialmente reservado para a visita, realizando-a apenas quando necessário.

A maioria dos médicos entrevistados $(75,0 \%)$ desenvolvia outras atividades médicas, além daquelas exercidas no PSF: $36,5 \%$ em serviços de emergência sob regime de plantão e em hospital, e 12,5\% em consultório particular e em ambulatório hospitalar. Entre os profissionais, $13,5 \%$ exerciam mais de uma atividade além daquelas desempenhadas no PSF.

No que diz respeito à relação médico-paciente, $85,4 \%$ dos médicos a consideraram diferenciada daquela exercida em outros contextos de atendimento, principalmente por ter uma maior proximidade e confiança $(74,0 \%)$. Outra mudança muito referida foi um maior comprometimento do médico e o tipo de atendimento no qual o paciente é visto integralmente $(21,9 \%)$.

Apesar dos dados do censo datarem do ano 2000, estes constituem um conjunto de informações relevantes porque refletem a realidade do início da implantação do PSF na Bahia.

\section{A relação médico-paciente ao vivo: análise observacional}

A análise observacional orientada por um instrumento estruturado enfoca a relação médico-paciente no tempo real da consulta, contemplando várias dimensões, tais como a in vestigação do problema e a formulação do plano terapêutico. Observaram-se 408 consultas ambulatoriais, a maioria tendo duração de 8 a 15 minutos.

De acordo com as observações orientadas pelo instrumento, a investigação do problema é conduzida principalmente mediante a anamnese biomédica e exames físicos, sem que sejam exploradas as condições de vida do paciente e confirmadas as hipóteses produzidas pela queixa principal. Neste sentido, não foi observada a explicitação diagnóstica, com esclarecimento do problema ao paciente, na maioria das consultas. Portanto, o diagnóstico, muitas vezes, não é discutido com o paciente, mantendo-o excluído da construção da compreensão do processo saúde-doença vivenciado, bem como da formulação do plano terapêutico, que implica procedimentos subsequentes à consulta e incorporação de novos comportamentos e atitudes de cuidado com a saúde. Esta constatação confronta a afirmação que atribui ao PSF uma mudança imediata na qualidade da relação entre profissionais de saúde e usuários.

A análise observacional aponta que a di mensão biomédica da consulta prevalece e que o momento da consulta não é aproveitado pelo médico para o desenvolvimento da consciência sanitária e da autonomia dos pacientes no cuidado com a própria saúde. A não abordagem da dimensão psicossocial, a exemplo da ansiedade dos pacientes frente ao problema de saúde, a condição de pobreza e de desemprego, caracterizam uma orientação marcadamente biomédica das consultas. Em algumas oportunidades, quando a queixa continha um fator psicossocial relevante, este não era incorporado à sua investigação e compreensão, o que foi observado para todos os médicos.

\section{A relação médico-paciente e as diretrizes do PSF: análise temática}

A análise temática possibilita uma compreensão da relação médico-paciente no contexto das diretrizes do PSF. Pretendeu-se, com esta proposta, uma análise da relação tendo como parâmetros elementos que visam a garantir a unidade e qualidade da estratégia do PSF.

\section{Natureza do PSF}

Observou-se a existência de uma modalidade do PSF para cada um dos três municípios estudados. As equipes do PSF do Município I não dispunham de um sistema de referência estruturado e de um programa de assistência farmacêutica. Para os pacientes, esta situação dificultava a execução do plano terapêutico pro posto, repercutindo na avaliação da resolutividade das ações. Esta situação influenciava negativamente na relação médico-paciente, uma vez que comprometia a própria continuidade da assistência.

O Município II possuía uma realidade em que as ações dos profissionais médicos, em geral, se dividiam em ações ocorridas no âmbito 
da unidade e ações comunitárias. Além disso, contava com uma rede de referência para realização dos exames complementares e das consultas especializadas, sendo prestada a assistência farmacêutica. Estas condições favoreciam o estabelecimento de vínculos entre médicos e pacientes que, por terem como combater os danos à saúde, tratavam de aspectos saudáveis, possibilitando ações na perspectiva da prevenção e promoção.

O Município III tinha uma realidade parecida com a do Município I no que se refere à organização do sistema e à ausência de assistência farmacêutica. Entretanto, o PSF era menos caracterizado porque não desenvolvia ações articuladas com os agentes comunitários de saúde (ACS), ações extramuros. Como muitos dos profissionais desse município mudaram seu regime de trabalho com a implantação do PSF, isso gerou diferenças nas rotinas principalmente no que diz respeito à carga-horária, mas nas práticas ainda verificavam-se elementos do modelo assistencial hegemônico.

O episódio, a seguir, exemplifica um aspecto da natureza do PSF na medida em que ilustra a possibilidade de acesso a outro nível de complexidade da atenção à saúde, viabilizado pela existência de uma rede de serviços de saúde local que faz funcionar o sistema de referência.

- Episódio 1

Médico (M): “No caso... dependendo disso, que pode ser uma coisa que a gente pode resolver aqui, uma coisa que for clínica ou, se a gente não puder resolver aqui, eu te encaminho pra uma ginecologista, certo? Uma colega da gente que trabalha [na zona urbana do município]".

\section{Vigilância da saúde}

A maioria dos episódios que contemplam aspectos da vigilância da saúde consiste em experiências de oportunidades não aproveitadas. Com esses episódios, pode-se afirmar que não sendo uma prática ouvir a história dos pacientes, a construção do raciocínio clínico-epidemiológico toma como informações dados gerais que compõem a situação de saúde da população e que não são investigados individualmente.

Três médicos observados no Município II demonstraram compreender a abordagem da vigilância da saúde. Estes episódios caracterizam-se pela construção da história clínica entre médico e paciente, tornando-se possível sinalizar ao paciente aspectos das condições de vida que influenciavam na queixa, possibili tando estratégias de enfrentamento destas condições que, tendo sido construídas conjuntamente, ao invés de prescritas, possivelmente favoreciam o desenvolvimento da autonomia do paciente.

No Município I, observou-se que os pacientes freqüentemente chegavam especificamente pedindo exames complementares, e que ao fim do ano solicitavam check-up. A população demonstrava não conhecer os princípios do PSF. Em paralelo, por se tratar de um município localizado na região metropolitana, a população parecia ser mais conhecedora da medicina tecnológica e a solicitava, invertendo os objetivos da abordagem da vigilância da saúde. Tal diferencial configurava um desafio para os profis sionais das equipes.

A superficialidade da compreensão da vigilância da saúde observada entre a maioria dos médicos leva a pensar que se trata também de um aspecto da formação acadêmica. O questionamento diante desta consideração seria: qual a justificativa para ouvir a história do paciente e construir com este o raciocínio clínico-epidemiológico fundamental para tomada de decisões no contexto do PSF? A condição técnica do médico é considerada suficiente para a tomada de decisões e não se justifica que tais decisões sejam partilhadas com os pacientes.

Nas experiências de oportunidades não aproveitadas, o médico não orienta sua ação pelos princípios da vigilância da saúde, trabalhando na lógica da demanda espontânea, sem atuação sobre os riscos, mantendo-se dono do saber e autoridade da saúde.

O médico do episódio abaixo trabalha numa unidade que atende a uma comunidade onde várias mães referiam fazer uso da farinha como engrossante do mingau das crianças. A despeito desta realidade, o médico não explorava os recursos que os pacientes dispunham para o cuidado com a saúde, inclusive para a sua alimentação, e prescrevia a dieta listando alimentos que deveriam ser consumidos pela comunidade.

- $\quad$ Episódio 2

M: "Nem açúcar, nem sal, tem que diminuiro máximo. Frutas, todas as frutas e os legumes também, viu? E verduras, legumes e verduras, todas as verduras, vai botar é cenoura, batata, chuchu, abóbora, beterraba... não bote tudo de vez que a gente não vai fazer um cozido [risos], mas você vai variando uma abobrinha, inhame, tudo isso você pode usar na comida dela, carne magra... Que é carne magra? Carne de boi, o frango...”.

Paciente (P): "Frango desfiado ela come na sopa".

M: "Carne magra de preferência, como já disse, na comida açúcar e sal pode tirar o máximo que puder, e fígado também, viu? Pode fazer fígado umas duas vezes na semana, viu?". 


\section{Compromisso com a população adscrita}

O compromisso com a população, compreendido como a co-responsabilização da equipe pelos problemas e pelas famílias da área adscrita, assume diversas configurações. As diferenças relacionam-se com a compreensão do PSF vivenciada por cada equipe. Os médicos mais comprometidos eram os que atuavam na zona rural. Nestes, vê-se facilmente uma série de ações que extrapolam as ações de planejamento e de atenção, indicando uma concepção ampliada de saúde.

Pôde-se observar médicos muito ativos, demonstrando proximidade com a população, mas este perfil não constitui necessariamente compromisso. O profissional, ao se colocar como um cumpridor de suas tarefas e ao fazer recomendações à população, sem contemplar características da realidade local, não exercitava o compromisso previsto pelas diretrizes do PSF. As condições de trabalho não favoreciam a organização da demanda e esta era dificultada porque o propósito do profissional era desincumbir-se da tarefa. Formas enfáticas de prescrever aparentemente eram tidas como compromisso, responsabilidade, mas as pessoas atendidas não eram distinguidas, moradores de outras áreas e de outros municípios eram atendidos na unidade.

A seguir, apresenta-se um episódio ilustrativo do compromisso com a população coerente com as diretrizes do PSF. Trata-se de um médico que atendia na zona rural. A equipe se comprometia em marcar os exames e consultas que precisavam ser realizados na zona urbana. Os pacientes, que em sua maioria tinham dificuldade com o dinheiro para o transporte, recebiam do ACS a requisição do procedimento agendado e eram orientados quanto ao dia e local de sua realização.

- Episódio 3

M: “Esse exame aqui (...), exame de coração, a gente leva pra [zona urbana], certo? Nós vamos marcar lá, ai depois o exame volta pr'aqui marcado".

P: "Sei".

M: "Aí o senhor com uma semana mais ou menos, talvez...é, segunda-feira...(...). Aí aqui vai dizer o dia, a data, né? No caso, e o local onde o senhor vai fazer. Aí no outro dia... não, segunda-feira o senhor já pode perguntar...".

P: "Eu pergunto".

M: “...pra [o agente], viu, se já veio ou não veio".

\section{Trabalho em equipe}

Com relação ao desenvolvimento do trabalho, nos Municípios I e II observou-se uma mudança da rotina do médico em decorrência de um trabalho complementar com o ACS. Principalmente no Município I, os ACSs eram muito referidos. Não se pode afirmar a existência de trabalho em equipe, porém a importância dos ACSs é reconhecida pelo médico do PSF e a sua presença nas equipes possibilitou mudanças no atendimento à população.

Circunstâncias ou falas em que o médico se refere à participação de outros integrantes da equipe no processo saúde-doença-cuidado exemplificam o reconhecimento do trabalho em equipe.

\section{- Episódio 4}

M: "Quando você tiver com ele [o exame] pronto, você tem que ver um agente, o seu agente tá de licença maternidade, pra você saber qual o horário que eu vou tá atendendo pra você não vir sozinha, arriscar, viu? Que às vezes eu tô na comunidade...".

$$
\text { P: “Tá bom”. }
$$

\section{Família como unidade de trabalho}

Todos os médicos compreendem o conceito de família como agrupamento humano constituído por laços de consangüinidade. Algumas equipes ampliam esta compreensão, porém não o fazem de forma sistemática. A família não parece ter sido assimilada como unidade de trabalho, sendo sistematicamente referida para tratar dos antecedentes mórbidos. Em algumas equipes, é vista como unidade de organização e objeto de atenção quando se trata de doenças infectocontagiosas. Os familiares não são considerados como co-responsáveis pelas ações do serviço de saúde, quando se trata de fortalecer estratégias de cuidado.

Entre os médicos estudados, apenas um do Município I demonstrou interesse pela estrutura e dinâmica familiar, mas as informações coletadas ou disponibilizadas espontaneamente não geravam ações específicas.

A família deixa de ser considerada como unidade de atenção e cuidado quando mais de um de seus membros estão presentes na consulta, mas o atendimento restringe-se àquele que tinha consulta marcada, enfatizando-se o atendimento individual. Na situação abaixo, o médico não considera a família como objeto e/ou parceiro na atenção, cuidado e resolução de problemas.

- Episódio 5

M: "Hum, hum, certo. O ideal era fazer um... assim primeiro você tem que examinar, né? Tem 
que ter o momento assim próprio para ele [o filho]. Certo? Se cada pessoa que entra traz dois problemas aí a gente marca dez consultas...".

P: "É isso mesmo".

M: “...que são vinte, né?”.

\section{Característica da atenção}

As condições dos municípios distinguem a atenção prestada pelos profissionais. Os Municípios I e III, por não possuírem rede de referência e assistência farmacêutica estruturadas, não podiam garantir a continuidade da assis tência, prejudicando também a resolutividade. Nestas equipes, a assistência do PSF era associada ao regime de trabalho do médico e a disponibilidade decorrente. Especialmente para um médico do Município III, a condição da falta de medicamentos interferiu nas suas rotinas, com esvaziamento da unidade.

Os médicos do Município II, guardando diferenças de estratégias de atuação, têm o exercício da atenção marcado pela continuidade e resolutividade, contando com consultas e visitas domiciliares, integralidade que abrange a saúde bucal atendida na unidade, rede de serviços especializados, racionalidade, considerando a priorização da assistência e a hierarquização desta, tendo o PSF como "porta de entrada" para o sistema de saúde.

Assistência integral, contínua, resolutiva e racionalizada especificam características da atenção. O episódio abaixo ilustra continuidade da assistência.

- Episódio 6

P: “E também eu sinto uma dor muito forte na minha barriga. Mas isso não é direto, né, é de vez em quando".

M: "Você já teve esse problema, lembra? Ano passado, no início do ano passado".

P: "Que eu vim aqui?".

M: "Sim, não foi comigo mas foi com o outro colega, que na ficha tá...”.

P: “Ah! Sei, sei, isso mesmo, acho que era lá no posto, né? (...) Aí acho que me pediu exames de fezes, aí parece que eu fiz e não deu nada nessa época não".

M: "E você fez preventivo também na época...". P: "Isso, isso mesmo".

\section{Paciente como sujeito}

Compreende-se que o paciente é reconhecido como sujeito quando tomado como agente do processo saúde-doença-cuidado, buscando desenvolver sua autonomia, co-responsabilidade, distinguindo no problema o contexto pessoal, familiar e social. O paciente enquanto su- jeito era uma realidade observada entre médicos dos Municípios I e II. Observou-se que as histórias eram ouvidas, as iniciativas de cuidados anteriores às consultas eram respeitadas e, na medida do possível, valorizadas. Principalmente com relação aos pacientes crônicos eram aproveitadas oportunidades para o desenvolvimento da autonomia, com a utilização de es tratégias como, por exemplo, simulação de situações e procedimentos na aplicação de insulina. Os pacientes participavam da formulação do plano terapêutico e tomavam decisões sobre alternativas de cuidados.

No Município III, foram observados episódios de desqualificação da história do paciente, das alternativas de cuidados construídas autonomamente, dos comportamentos, principalmente quando se tratava de comportamentos sexuais julgados por princípios morais, não necessariamente compartilhados pelos pacientes.

No episódio abaixo o médico utiliza da estratégia de simulação, por meio da qual se pode observar que o desenvolvimento da autonomia do paciente é estimulada, e ele se sente responsabilizado por sua saúde.

\section{- $\quad$ Episódio 7}

M: “Quem aplica a insulina?".

Acompanhante (AC): "Eu mesmo".

M: “A senhora?”.

AC: "É".

M: “Certo. Vamos ver aqui. (...) Como é que a senhora aplica a insulina? Faz de conta que isso aqui é insulina e a senhora vai aplicar nele agora".

AC: “Eu faço isso, né? Puxa um pouquinho... puxa um pouquinho para ver se tem sangue. Depois aplico".

M: "A agulha perpendicular, né? Ou seja, não pode ser assim nem assim. E depois de usar o que é que faz com a seringa?".

AC: "Aí é porque ele pediu para usar dois dias ou três e depois jogar fora. Porque é uma pessoa só".

M: “É, não é necessário jogar fora, já que é ele só que usa, né?”.

AC: "Tem que trocar a agulha".

M: "É tem que trocar a agulha só. Mas a seringa, parte de baixo, não é necessário, pode usar mais de uma vez. Tá certo? Coloca a insulina na geladeira. Se vier sangue não pode aplicar".

AC: "Não pode aplicar".

M: “Essa seringa não pode ser reutilizada. Se, por acaso, a senhora puxar e vier sangue tem que jogar essa fora. Por quê? O sangue coagula, entope a seringa, aí não pode. Mas se não vier sangue não tem problema. Troca a agulha e toma o remédio, tá certo? Quais são as partes do 
corpo, não aplicar sempre na mesma área, todo... repetido. Mudar as áreas do corpo, né?".

A análise temática propiciou a identificação de alguns desafios na relação médico-paciente frente à proposta apresentada nas diretrizes do PSF.

\section{Caracterizando a relação médico-paciente: análise interacional}

A caracterização da relação médico-paciente possibilitou a elaboração de cinco categorias interacionais, contemplando aspectos orientados pelo macrocontexto, que seriam elementos presentes nas diretrizes do PSF: compreensão integral do paciente, desenvolvimento da autonomia do paciente, prevenção da doença e promoção da saúde. Duas categorias interacionais se centram na estrutura microcontextual da relação, privilegiando aspectos comunicacionais: extensão das falas e tipos de pergunta/resposta. Este nível de análise acentua os resultados anteriores.

A compreensão integral do paciente não foi observada enquanto característica da relação médico-paciente, predominando uma escuta acentuadamente biomédica da queixa e uma ação prescritiva. Quando os relatos dos paciente apresentavam conteúdos que possibilitariam aprofundamento de aspectos psicossociais, estes freqüentemente não eram valorizados pelo médico.

O desenvolvimento da autonomia pode se dar na articulação da história do paciente com o quadro clínico e a construção conjunta dos meios para lidar com as situações. Em geral, essa articulação não foi verificada.

Conjuntamente com o modo de compreensão integral do paciente e o desenvolvimento da autonomia, a prevenção da doença e a promoção da saúde não eram incorporadas nas práticas de três dos quatro médicos analisados. O trabalho médico era orientado pelo eixo queixas - exames laboratoriais - prescrições, sendo os exames distinguidos pelos profissionais como prática preventiva. O médico que incorporava a prevenção e a promoção em sua prática explorava a avaliação da saúde feita pelo paciente, sugerindo o que poderia ser feito e solidarizando-se com perdas ocorridas decorrentes das restrições vinculadas ao comportamento preventivo.

As categorias relativas à comunicação médico-paciente estão articuladas entre si. A extensão das falas do médico associa-se ao tipo de pergunta formulada. Observou-se uma predominância de falas sintéticas dirigidas para a queixa, buscando-se a confirmação da hipótese diagnóstica, com freqüentes interrupções às falas dos pacientes. Nesta interação, a comunicação tem a imagem de um chek-list, com perguntas diretivas, fechadas, podendo ser respondidas com uma única afirmação.

A relação médico-paciente reconhecida como mais coerente com a proposta da estratégia do PSF, contemplaria a formulação de perguntas abertas utilizando-se como estratégia a repetição da fala do paciente para enfatizar ou questionar situações relatadas. Dentre os quatro médicos analisados, apenas um apresentou este modo de agir. Este profissional atuava no PSF na zona rural do Município II. Tal condição possibilita questionar se as caraterísticas contextuais (zona rural do Município II) seriam favorecedoras deste modo de interação.

\section{Considerações finais}

Este estudo adotou estratégias de coleta e análise de dados que fossem sensíveis à observação de aspectos micro e macrocontextuais incidentes sobre a relação médico-paciente.

Um elemento sintetizador que distingue os municípios estudados, e possivelmente o PSF de outras estratégias de atenção baseadas na unidade familiar, é a abordagem da vigilância da saúde. Esta, integra prevenção da doença e promoção da saúde à participação e ao controle social. Os resultados demonstram que, quanto mais se exercita os princípios da abordagem da vigilância da saúde, mais se está imprimindo uma prática inovadora no sistema de saúde.

Tomando o conjunto dos resultados obtidos, pode-se afirmar ainda que a proposta do PSF, quando executada, favorece uma relação médico-paciente diferenciada, marcada pelos princípios da vigilância da saúde, da continuidade, da integralidade, do respeito à condição do paciente como sujeito. Desta constatação depreende-se que a relação médico-paciente no PSF nos três municípios estudados sofre influências macro e microcontextuais.

Na singularidade do momento clínico, elementos macrocontextuais como a assistência farmacêutica e a existência de uma rede integrada e hierarquizada repercutem na relação médico-paciente ao comprometer a continuidade da assistência e, por conseguinte, do vínculo entre usuário e serviço. Por outro lado, compreende-se que o PSF, enquanto uma estratégia de reorientação do modelo assistencial, não vem garantindo uma diferenciação da relação médico-paciente, uma vez que a realidade microcontextual não se encontra sim- 
plesmente como receptora das influências macrocontextuais.

Usar os princípios da vigilância da saúde para guiar o trabalho clínico, dirigido para o individual ou para o familiar, é uma proposta que carece ainda de aprofundamento. Neste trabalho parece ter sido feita uma primeira tentativa. Os resultados sugerem que os médicos conhecem os fatores de risco e os exploram, mas não implicam os pacientes nisso, exercitando, deste modo, o modelo paternalista de atenção ou o modelo superficialmente biomédico 4,12.

A dimensão psicossocial, condição indis pensável à efetivação de uma atenção integral, precisa ainda ser incorporada à prática médica, não como uma espécie de acessório humanizador, mas enquanto componente essencial dessa mesma prática. A desqualificação ou a indiferença frente a dimensão psicossocial pode representar uma condição favorecedora à medicalização.

\section{Resumo}

Estudou-se a relação médico-paciente no Programa Saúde da Família (PSF). Compreendendo que esta sofre influência de fatores macro e microcontextuais, optou-se por uma articulação da abordagem da vigilância da saúde com o modelo ecológico do desenvolvimento humano, compreensões influenciadas pelo contextualismo. As estratégias metodológicas consistiram no censo dos médicos de saúde da familia da Bahia, Brasil; observações de consultas de vinte médicos de três municípios, orientadas por um instrumento. Os resultados indicam diferenças significativas entre a implementação do PSF nos três municípios, repercutindo na relação médico-paciente. Quanto à orientação das práticas, a vigilância da saúde não tem sido incorporada pelos médicos observados. A análise microcontextual apontou que os problemas de saúde freqüentemente não eram tratados de modo contextualizado. Compreende-se que as diretrizes do PSF, quando assumidas pelos municípios, são produtoras de diferenças na assistência observáveis no microcontexto da relação médico-paciente. A abordagem da vigilância da saúde, referida como ferramenta para o trabalho coletivo, mostra-se eficiente para descrever, avaliar e aperfeiçoar o PSF e, neste estudo, conclui-se que esta é uma ferramenta conceitual potencial para implementação da relação médico-paciente.

Relações Médico-Paciente; Programa Saúde da Família; Vigilância em Saúde Pública
Em síntese, os resultados do presente estudo apontam: (i) existem diferenças significativas entre os modos de implantação do PSF nos três municípios, repercutindo na relação médico-paciente; (ii) a continuidade da assistência foi compreendida nos vários níveis de análise de dados dependente da existência de uma rede de serviços de saúde hierarquizada a ser referenciada; e (iii) a vigilância da saúde não tem sido incorporada nas práticas dos médicos observados.

A abordagem da vigilância da saúde, referida como ferramenta para o trabalho coletivo, é ampla e sólida a ponto de descrever, avaliar e aperfeiçoar o PSF. Neste estudo foi reconhecida como uma ferramenta conceitual potencial para implementação da relação médico-paciente.

\section{Colaboradores}

A. L. S. Franco desenvolveu o projeto. Em conjunto com V. S. Alves realizaram a coleta, a análise de dados e a redação do texto. A. C. S. Bastos participou na orientação do projeto.

\section{Agradecimentos}

Ao Prof. Andrea Caprara, coordenador geral do estudo, pelas cuidadosas sugestões ao longo do trabalho. 


\section{Referências}

1. Ministério da Saúde. Saúde da família: uma estratégia para a reorganização do modelo assistencial. Brasília: Ministério da Saúde; 1997.

2. Silva LMV, Formigli VLA, Cerqueira MP, Kruchevsky L, Teixeira MMA, Barbosa ASM, et al. O processo de distritalização e a utilização de serviços de saúde - avaliação do caso de Pau da Lima, Salvador, Bahia, Brasil. Cad Saúde Pública 1995; 11:7284.

3. Ong LML, Haes JCJM, Hoss AM, Lammes FB. Doctor-patient communication: a review of literature. Soc Sci Med 1995; 40:903-18.

4. Roter DL, Stewart M, Putnam SM, Lipkin Jr. M, Stiles W, Inui TS. Communication patterns of primary care physicians. JAMA 1997; 277:350-6.

5. Levinson W, Roter DL, Mullooly J, Dull VT, Frankel RM. Physician-patient communication: the relationship with malpractice claims among primary care physicians and surgeons. JAMA 1997; 277:5539.

6. Williams J, Weinman J, Dale J. Doctor-patient communication and patient satisfaction: a review. Fam Pract 1998; 15:480-92.

7. Sucupira ACSL. Relações médico-paciente nas instituições de saúde brasileiras [Dissertação de Mestrado]. São Paulo: Universidade de São Paulo; 1981.
8. Bronfenbrenner U. A ecologia do desenvolvimento humano. Porto Alegre: Editora Artes Médicas; 1996.

9. Franco ALS, Bastos ACS. Um olhar sobre o Programa de Saúde da Família: a perspectiva ecológica na psicologia do desenvolvimento segundo Bronfenbrenner e o modelo da vigilância da saúde. Psicol Estud 2002; 7:65-72.

10. Caprara A, Bastos ACS, Franco ALS, Alves VSA, Rodrigues J, Braga JM, et al. A relação médico-paciente no Programa Saúde da Família: uma pesquisa-ação com as equipes de saúde da família do Ceará e da Bahia [Relatório final]. Fortaleza: Escola de Saúde Pública/Salvador: Instituto de Saúde Coletiva; 2003.

11. Grol R, Lawrence, M. Instruments for review of general consultation skills. Quality improvement by peer review. Oxford: Oxford University; 1995.

12. Emanuel EJ, Emanuel LL. Four models of the physician-patient relationship. JAMA 1992; 267:2221-6.

Recebido em 08/Mar/2004

Versão final reapresentada em 30/Jun/2004

Aprovado em 17/Ago/2004 